\title{
A Porism Concerning Cyclic Quadrilaterals
}

\author{
Jerzy Kocik \\ Department of Mathematics, Southern Illinois University, Carbondale, IL 62901, USA \\ Correspondence should be addressed to Jerzy Kocik; jkocik@siu.edu
}

Received 26 April 2013; Accepted 15 July 2013

Academic Editor: Michel Planat

Copyright (C) 2013 Jerzy Kocik. This is an open access article distributed under the Creative Commons Attribution License, which permits unrestricted use, distribution, and reproduction in any medium, provided the original work is properly cited.

We present a geometric theorem on a porism about cyclic quadrilaterals, namely, the existence of an infinite number of cyclic quadrilaterals through four fixed collinear points once one exists. Also, a technique of proving such properties with the use of pseudounitary traceless matrices is presented. A similar property holds for general quadrics as well as for the circle.

\section{Introduction}

Inscribe a butterfly-like quadrilateral in a circle and draw a line $L$, see Figure 1 . The sides of the quadrilateral will cut the line at four (not necessarily distinct) points. It turns out that as we continuously deform the inscribed quadrilateral, the points of intersection remain invariant.

More precisely, think of the quadrilateral as a path from vertex $X$ through collinear points $P, R, S$, and $Q$ back to $X$ (see Figure 4 , left). If we redraw the path starting from another point on the circle but passing through the same points on the line in the same order, the path closes to form an inscribed polygon; that is, we will arrive at the starting point.

In spirit, this startling property is similar to Steiner's famous porism $[1,2]$, which states that once we find two circles, one inner to the other, such that a closed chain of neighbor-wise tangent circles inscribed in the region between them is possible, then an infinite number of such inscribed chains exist (Figure 2). One may set the initial circle at any position and the chain will close with tangency. Yet, another geometric phenomenon in the same category is Poncelet's porism [3-5].

The property for the cyclic quadrilateral described at the outset may be restated similarly: if four points on a line admit a cyclic quadrilateral, then an infinite number of such quadrilaterals inscribed in the same circle exist. Hence, the term porism is justified.

In the next sections, we restate the theorem and define a map of reversion through point, which may be represented by pseudounitary matrices. The technique developed allows one to prove the theorem as well as a diagrammatic representation of the relativistic addition of velocities, presented elsewhere [6]. A slight modification to arbitrary two-dimensional Clifford algebras allows one to modify the result to hold for hyperbolas and provides a geometric realization of trigonometric tangent-like addition.

\section{The Main Result}

Let us present the result more formally. Reversion of a point $A$ on a circle through point $P$ gives point $\mathbf{P}(A)$ on the circle such that points $A, P$, and $\mathbf{P}(A)$ are collinear (see Figure 3 ). We can define it more precisely as follows.

Definition 1. Given a circle $K$, a reversion through point $P \notin$ $K$ is a map $\mathbf{P}: K \rightarrow K, A \mapsto \mathbf{P}(A)$, such that points $A, P$, and $\mathbf{P}(A)$ are collinear and $\mathbf{P}(A) \neq A$. If $P \in K$, then for any $A \in K$, one defines $\mathbf{P}(A)=P$.

By bold $\mathbf{P}$, we denote the reversion map defined by point $P$ (not bold). Clearly, reversion through $P \notin K$ is an involution, $\mathbf{P}^{2}=\mathrm{id}$, and therefore invertible. The product of reversions is in general not commutative, $\mathbf{P Q} \neq \mathbf{Q P}$. The main result may now be given a concise form as follows.

Theorem 2. Let $P, Q, R$, and $S$ be collinear points, $K a$ circle, and $\mathbf{Z}=\mathbf{S R Q P}$ the composition of the corresponding reversions. Then,

$$
\text { if } \exists X \in K \quad \mathbf{Z}(X)=X, \text { then } \forall X \in K \quad \mathbf{Z}(X)=X \text {. }
$$



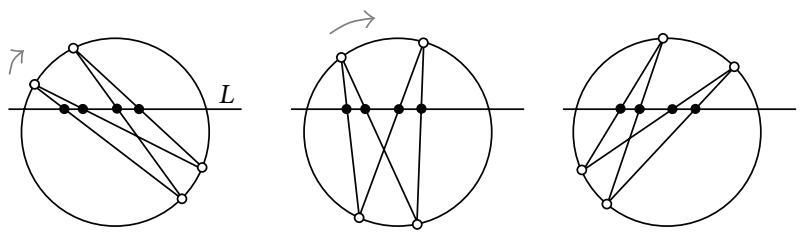

Figure 1: Porism on concyclic quadrilaterals through collinear points.

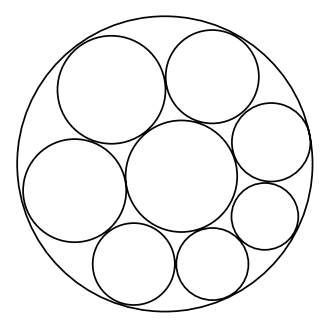

FIGURE 2: Steiner's porism. The chain may be redrawn starting from arbitrary position of the starting circle.

The quadrilateral $[X, \mathbf{P}(X), \mathbf{Q P}(X), \mathbf{R Q P}(X)]$ may be viewed as a member of a family parametrized by $X$. As $X$ moves along the circle, the quadrilateral continuously changes its form while the points of intersection with the line remain invariant. The quadrilateral appears to be "rotating" with points $P, Q, R$, and $S$ playing the role of "axes" of this motion. For an animated demonstration, see [7].

Here is an equivalent version of Theorem 2.

Theorem 3. Let $K$ be a circle and $L$ a line with three points $P, Q$, and $R$. Let $X$ be a point on the circle. The point $S$, an intersection of line $L$ with line $[\mathbf{R Q P}(X), X]$, does not depend on $X \in K$.

In other words, for any three collinear points $P, Q$, and $R \in L$, there exists a unique point $S$ on $L$ such that any cyclic quadrilateral inscribed in $K$ passing through $R, P$, and $Q$ (in the same order) must also pass through $S$.

A more general statement holds.

Theorem 4. The composition of three point reversions $\mathbf{P Q R}$ of a circle is a reversion if and only if points $\mathbf{P}, \mathbf{Q}$, and $\mathbf{R}$ are collinear.

Remark 5. The figures present quadrilaterals in the butterfly shape for convenience. Clearly, they can have untwisted shape, and also, the points of intersection can lie outside the circle, and the line does not need to intersect the circle.

\section{Reversion Calculus-Matrix Representation}

In order to prove the theorem, we develop a technique that uses complex numbers and matrices. Interpret each point $P$ as a complex number $p \in \mathbb{C}$. Without loss of generality, we will assume that $K$ is the unit circle, $K=\left\{z \in \mathbb{C}:|z|^{2}=\right.$ $1\}$. Complex conjugation is denoted in two ways. Here is our main tool.

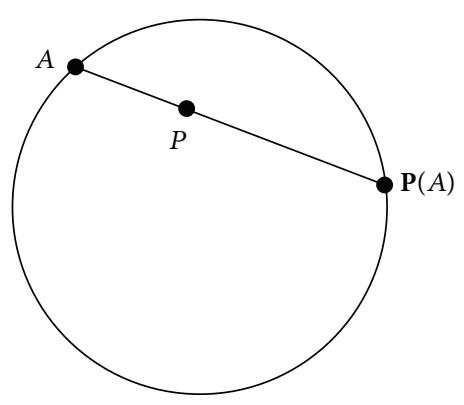

Figure 3: Reversion of $A$ through $P$.
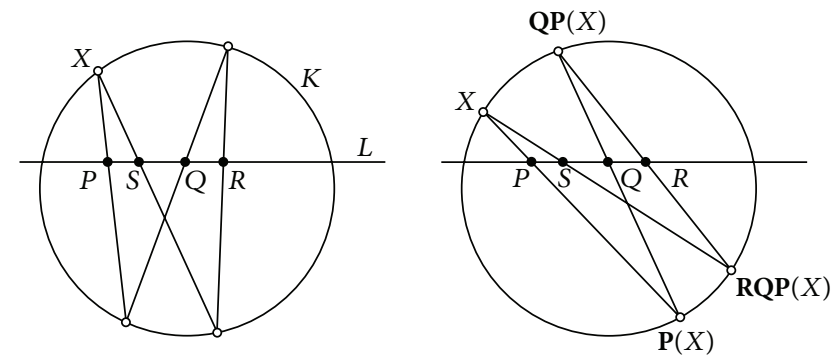

Figure 4: Porism: details.

Theorem 6. Reversion in the unit circle $K$ through point $p \in \mathbb{C}$ corresponds to a Möbius transformation:

$$
\widehat{P}: z \longrightarrow z^{\prime}=\left[\begin{array}{ll}
1 & -p \\
p & -1
\end{array}\right] \cdot z=\frac{z-p}{\bar{p} z-1} .
$$

Proof. First, we check that reversions leave the unit circle invariant, $|z|^{2}=1 \Rightarrow\left|z^{\prime}\right|^{2}=1$ :

$$
\begin{aligned}
\left|z^{\prime}\right|^{2} & =\frac{z-p}{\bar{p} z-1}\left(\frac{z-p}{\bar{p} z-1}\right)^{*} \\
& =\frac{|z|^{2}-z \bar{p}-\bar{p} z+|p|^{2}}{|p|^{2}|z|^{2}-\bar{p} z-\bar{z} p+1} \\
& =\frac{1-z \bar{p}-\bar{p} z+|p|^{2}}{|p|^{2}-\bar{p} z-\bar{z} p+1}=1 .
\end{aligned}
$$

Next, we show that for any $z \in K$, points $\left(z, p, z^{\prime}\right)$ are collinear. We may establish that by checking that $\left(z^{\prime}-p\right)$ differs from $(z-p)$ only by scaling by a real number. Indeed, take their ratio:

$$
\begin{aligned}
\frac{z^{\prime}-p}{z-p} & =\frac{(z-p) /(\bar{p} z-1)-p}{z-p}=\frac{(z-p)-p(\bar{p} z-1)}{(z-p)(\bar{p} z-1)} \\
& =\frac{1-|p|^{2}}{\bar{p} z+p \bar{z}-|p|^{2}-1}=\frac{1-|p|^{2}}{2 \operatorname{Re}(\bar{p} z)-|p|^{2}-1} \in \mathbb{R}
\end{aligned}
$$

where, to get the first expression in the second line, we multiplied the numerator and the denominator by $\bar{z}$ and used the fact that $|z|^{2}=1$. It is easy (and not necessary) to check that $\widehat{P}^{2}=$ id (up to Möbius equivalence). 
A more abstract definition of the matrix representation emerges. Namely, denote the pseudounitary group of two-bytwo complex matrices:

$$
U(1,1)=\left\{A \in \mathbb{C} \otimes \mathbb{C} \mid A^{*} J A=J\right\},
$$

where $J$ denotes the diagonal matrix $J=\operatorname{diag}(1,-1)$, and the star "*” denotes usual Hermitian transpose. Let $\sim$ be the equivalence relation among matrices: $A \sim B$, if there exists $\lambda \in \mathbb{C}, \lambda \neq 0$, such that $A=\lambda B$. Then, we have a projective version of the pseudounitary group:

$$
P U(1,1)=U(1,1) / \sim,
$$

which is equivalent to its use for Möbius transformations. Now, any element of the group may be represented by a matrix up to a scale factor. The essence of Theorem 2 is that reversions correspond to such matrices with vanishing trace, $\operatorname{Tr} A=0$.

Note that we do not follow the tradition of normalizing the determinants, as is typically done for the representation of the modular group $\operatorname{PSL}(2, \mathbb{Z})$.

\section{Algebraic Proof of the Porism}

We will now prove the main results.

Proof of the Main Theorem. Consider the product of three consecutive reversions through points $P, Q$, and $R$, as represented by matrices

$$
\begin{aligned}
M & =\left[\begin{array}{ll}
1 & -r \\
\bar{r} & -1
\end{array}\right]\left[\begin{array}{ll}
1 & -q \\
\bar{q} & -1
\end{array}\right]\left[\begin{array}{cc}
1 & -p \\
\bar{p} & -1
\end{array}\right] \\
& =\left[\begin{array}{ll}
1-\bar{p} q+\bar{q} r-r \bar{p} & -p+q-r+p \bar{q} r \\
\bar{p}-\bar{q}+\bar{r}-\bar{p} q \bar{r} & -1+p \bar{q}-q \bar{r}+\bar{r} p
\end{array}\right] .
\end{aligned}
$$

Note that $M_{12}=-\bar{M}_{21}$, but we still need to see whether $\operatorname{Tr} M=0$. Recall the assumption that $p, q$, and $r$ are collinear. If $r=m p+n q$, where $m+n=1, m, n \in \mathbb{R}$, we observe that the diagonal elements are real:

$$
\begin{gathered}
M_{11}=1-m(\bar{p} q+p \bar{q})+m|p|^{2}-n|q|^{2} \in \mathbb{R}, \\
M_{22}=-M_{11} .
\end{gathered}
$$

Dividing every entry by $M_{11}$ (Möbius equivalence), we arrive at

$$
M=\left[\begin{array}{cc}
1 & \frac{-p+q-r+p \bar{q} r}{1-\bar{p} q-\bar{q} r+r \bar{p}} \\
\overline{\bar{p}-\bar{q}+\bar{r}-\bar{p} q \bar{r}} & -1
\end{array}\right],
$$

which is visibly a matrix of reversion, defining uniquely the point of reversion:

$$
s=\frac{p-q+r-p \bar{q} r}{1-\bar{p} q-\bar{q} r+r \bar{p}} .
$$

What remains is to verify that $s$ is collinear with $p$ and $q$ (and therefore $r$ ). This may be done algebraically by checking that

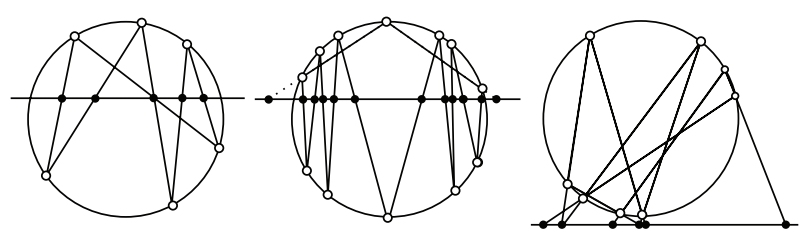

FIgURE 5: A porism of cyclic polygons.

$(s-p) /(r-q) \in \mathbb{R}$, but it also follows neatly by geometry that there are two points on the circle collinear with $P, Q$, and $R$, say $A$ and $B$. Since $\mathbf{R Q P}(A)=B$, and therefore, $\mathbf{S}(A)$ must be $B$, thus $S$ lies on the same line. We have proved that given a circle $K$, for any three points $P, Q$, and $R$, on a line $L$, there exists a point $S$ on $L$ such that $\mathbf{S P Q R}=$ id or $\mathbf{S}=\mathbf{P Q R}$, because $S^{2}=$ id. The main theorem follows.

The theorem generalizes to cyclic $n$-gons (see Figure 5).

Theorem 7. Let $\mathbf{P}=\mathbf{P}_{2 n} \mathbf{P}_{2 n-1}, \ldots, \mathbf{P}_{2} \mathbf{P}_{1}$ be the composition of point reversions of a circle $K$ for some collinear set of an even number of (not necessarily distinct) points $\mathbf{P}_{1}, \ldots, \mathbf{P}_{2 n}$. Then,

$$
(\exists X \in K, \mathbf{P}(X)=X) \Longrightarrow(\forall X \in K, \mathbf{P}(X)=X) .
$$

Proof. By Theorem 4, any three consecutive maps in the string $\mathbf{P}$ may be replaced by one. This reduces the string of an even number of reversions to a string of four, which is the case proven as Theorem 2. Further reduction to two reversions would necessarily produce $\mathbf{P}_{1} \mathbf{P}_{1}=\mathrm{id}$.

Remark 8. Theorem 7 suggests a concept of a "ternary semigroup of points" on a (compactified) line, where the product requires three elements to produce one:

$$
a, b, c \longrightarrow a b c .
$$

The following may be considered as "axioms":

(1) $(a b c) d e=a(b c d) e=a b(c d e)$ (associativity),

(2) $a b b=b b a=a$ (absorption of squares),

(3) $a b c=c b a$ (mirror inversion of triples).

The point reversions define a realization of this algebra.

Yet another concept comes from these notes: two pairs of points, $(P, Q)$ and $(S, R)$, on a line are conjugate with respect to a circle if they belong to inscribed angles based on the same chord (see Figure 4, left), in other words, if $\mathbf{P Q}=\mathbf{S R}$, or, if there exists an inscribed quadrilateral which intersects $L$ in $P, Q, R$, and $S$. In matrix terms,

$$
\begin{aligned}
M(q) M(p) & =\left[\begin{array}{cc}
1-q \bar{p} & q-p \\
\bar{q}-\bar{p} & 1-\bar{q} p
\end{array}\right] \\
& =\left[\begin{array}{cc}
1 & \frac{q-p}{1-q \bar{p}} \\
\frac{\bar{q}-\bar{p}}{1-q \bar{p}} & \frac{1-\bar{q} p}{1-q \bar{p}}
\end{array}\right]=\left[\begin{array}{cc}
1 & \frac{q-p}{1-q \bar{p}} \\
\left(\frac{q-p}{1-\bar{q} p}\right)^{*} & \frac{1-\bar{q} p}{1-q \bar{p}}
\end{array}\right],
\end{aligned}
$$




$$
\begin{aligned}
M(r) M(s) & =\left[\begin{array}{cc}
1-r \bar{s} & r-s \\
\bar{r}-\bar{s} & 1-\bar{r} s
\end{array}\right] \\
& =\left[\begin{array}{cc}
1 & \frac{r-s}{1-r \bar{s}} \\
\frac{\bar{r}-\bar{s}}{1-r \bar{s}} & \frac{1-\bar{r} s}{1-r \bar{s}}
\end{array}\right]=\left[\begin{array}{cc}
1 & \frac{r-s}{1-r \bar{s}} \\
\left(\frac{r-s}{1-\bar{r} s}\right)^{*} & \frac{1-\bar{r} s}{1-r \bar{s}}
\end{array}\right],
\end{aligned}
$$

from which this convenient formula results:

$$
\frac{r-s}{1-r \bar{s}}=\frac{q-p}{1-q \bar{p}}
$$

provided $p, q, r, s$ are collinear.

\section{Application: Relativistic Velocities}

Take the real line and the unit circle as ingredients for the model. Consider a quadrilateral that goes through three collinear points, the origin (0) and two points represented by real numbers $a$ and $b$. From Theorem 3, it follows that the fourth point on the real line must be

$$
\begin{aligned}
M(b) & M(a) M(0) \\
& =\left[\begin{array}{ll}
1 & -b \\
b & -1
\end{array}\right]\left[\begin{array}{ll}
1 & -a \\
a & -1
\end{array}\right]\left[\begin{array}{cc}
1 & 0 \\
0 & -1
\end{array}\right] \\
& =\left[\begin{array}{cc}
1+a b & -a-b \\
a+b & -1-a b
\end{array}\right] \sim\left[\begin{array}{cc}
1 & -\frac{a+b}{1+a b} \\
\frac{a+b}{1+a b} & -1
\end{array}\right] .
\end{aligned}
$$

We used the fact that conjugation does nothing to real numbers. Thus, the fourth point on $\mathbb{R}$ has coordinate

$$
a \oplus b=\frac{a+b}{1+a b},
$$

but this happens to be the formula for relativistic addition of velocities! (in the natural units in which the speed of light is 1). Thus, we obtain its geometric interpretation, presented in Figure 6. A conventional derivation of this diagram may be found in [6]. See also [7] for an interactive applet.

The segment through the origin does not have to be vertical for the device to work (by Theorem 3) but is set so for simplicity.

\section{Further Generalizations}

The porism described here for circles is also valid for any quadric; see Figure 7. What is intriguing, three cases (viz. circle, ellipse, and a pair of parallel lines) correspond to the three possible 2-dimensional Clifford algebras, each of the form of "generalized number plane" $\{a+b e\}$, where $e$ is an "imaginary unit" whose square is $1,-1$, or 0 , (see Table 1 ).

The case of the circle corresponds to complex numbers, as described in the previous sections. The case of the hyperbola corresponds to "duplex numbers," called also hyperbolic numbers or split-complex numbers:

$$
\mathbb{D}=\left\{a+b I: a, b \in \mathbb{R}, I^{2}=1\right\} .
$$

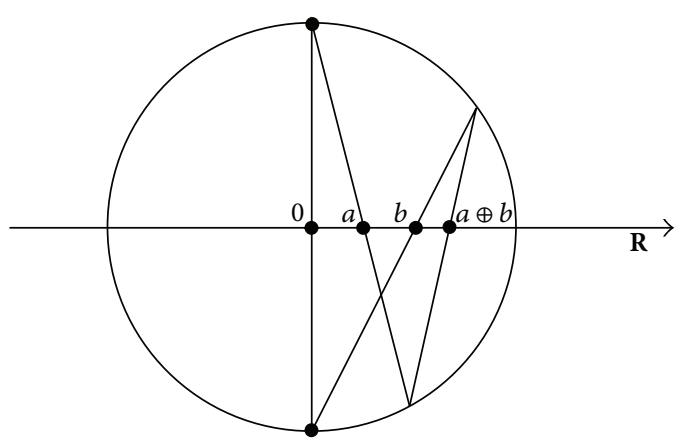

FIGURE 6: Relativistic addition of velocities.
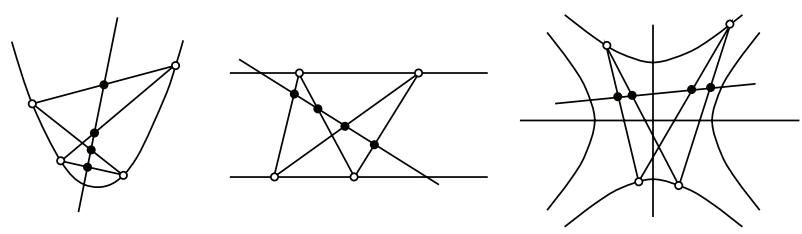

FIgURE 7: Porism on quadrilaterals inscribed in quadrics.

TABLE 1

\begin{tabular}{lcc}
\hline Algebra & “Sphere" & $\begin{array}{c}\text { Formula represented } \\
\text { geometrically }\end{array}$ \\
\hline $\mathbb{C}$ (complex numbers) & Circle & Relativistic velocities \\
$\mathbb{D}$ (hyperbolic numbers) & Hyperbola & $\begin{array}{c}\text { Trigonometric tangents } \\
\mathbb{G} \text { (dual numbers) }\end{array}$ \\
\hline
\end{tabular}

They are-like complex numbers - a two-dimensional unital algebra except that its "imaginary unit" $I$ is 1 when squared. As complex numbers are related to rotations, duplex numbers correspond to hyperbolic rotations. They were introduced in [8] as tessarines and represent a Clifford algebra $\mathbb{R}_{0,1}$. They found useful applications; for example, in [9], a "hyperbolic quantum mechanics" was introduced. Here is the theorem corresponding to Theorem 6 .

Proposition 9. The reversion through a point $p \in \mathbb{D}$ with respect to the hyperbola $x^{2}-y^{2}=1$ is represented by the matrix

$$
\widehat{p}: z \longrightarrow z^{\prime}=\left[\begin{array}{cc}
1 & -p \\
\bar{p} & -1
\end{array}\right] \cdot z=\frac{z-p}{\bar{p} z-1} \quad\left(|z|^{2}=1\right),
$$

while for branch $x^{2}-y^{2}=-1$, it is

$$
\widehat{p}: z \longrightarrow z^{\prime}=\left[\begin{array}{cc}
-1 & p \\
\bar{p} & 1
\end{array}\right] \cdot z=\frac{-z+p}{\bar{p} z+1} \quad\left(|z|^{2}=-1\right) .
$$

Proof. For (18), follow the lines of the proof of the main theorem. To get (19), multiply the ingredients of (18) by $I$ to switch the axes, use (18), and then multiply by $I$ again to restore the original axes.

For the last case of parallel lines, we can use the same previous matrix calculus, but replace the algebra by dual numbers

$$
\mathbb{G}=\left\{a+b I: a, b \in \mathbb{R}, I^{2}=0\right\} .
$$




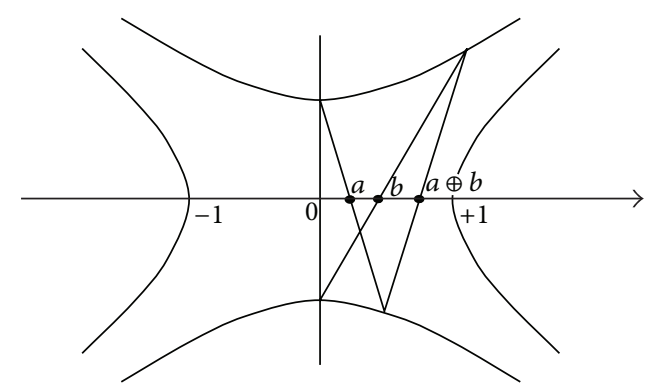

FIGURE 8: Trigonometric tangent formula visualized.
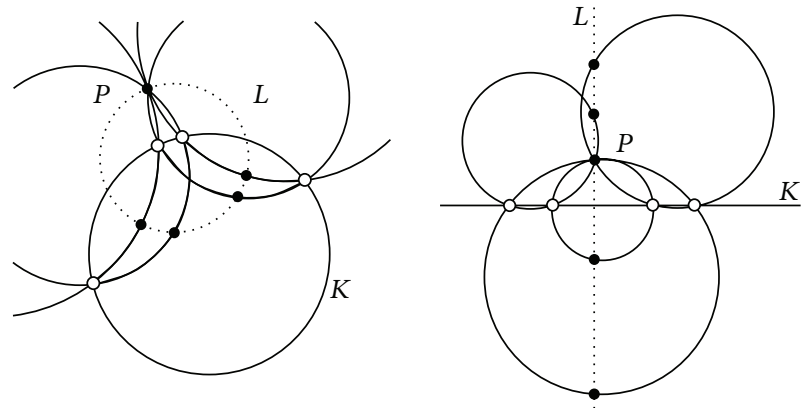

FIgure 9: Porism under inversion extends to circles replacing lines and vice versa.

Transformations (18) and (19) apply for two cases: vertical and horizontal lines, respectively $\left(|z|^{2}=1\right.$ versus $|z|^{2}=-1$ in the hyperbolic norm).

It is a rather pleasant surprise to find the algebra and geometry interacting at such a basic level in an entirely nontrivial way. Repeating a construction analogous to the one in Section 5 that results in a geometric tool for addition of relativistic velocities to the hyperbolic numbers gives a similar geometric diagram representing addition of trigonometric tangents; see Figure 8. Consider

$$
a \oplus b=\frac{a+b}{1-a b}=\tan (\arctan a+\arctan b) .
$$

The final comment concerns the obvious extension of these results implied by inversion of the standard configuration like in Figure 1 through a circle. Möbius geometry removes the distinction between circles and lines. The lines of the quadrilateral and the line $L$ under inversion may become circles. The point at infinity, where these lines meet, becomes under inversion a point that is part of the porism's construction.

Thus, the general statement is the following: given circles $L$ and $K$ and an odd number of points $P, P_{1}, P_{2}, \ldots, P_{2 n}$ on $L$, suppose that there exists an ordered $2 n$-tuple (pencil) of circles $C_{1}, \ldots, C_{2 n}$, through $P$ such that $P_{i} \in C_{i}$ for every $i$ and the points of intersections $C_{i} \cap C_{i+1}$ and $C_{2 n} \cap C_{1}$ different from $P$ all lie on the circle $K$; then, there are infinitely many such $2 n$-tuples of circles. Figure 9 illustrates the theorem for four circles. For interactive version of these and more examples, see [7].

\section{Acknowledgments}

The author is grateful to Philip Feinsilver for his interest in this work and for his helpful comments. Special thanks go also to creators of Cinderella, a wonderful software that allows one to quickly test geometric conjectures and to create nice interactive applets.

\section{References}

[1] D. Coxeter and S. Greitzer, Geometry Revisited, Mathematical Association of America, Washington, DC, USA, 1967.

[2] E. W. Weisstein, "Steiner Chain," From Math World-A Wolfram Web Resource, http://mathworld.wolfram.com/SteinerChain .html.

[3] W. Barth and T. Bauer, "Poncelet theorems," Expositiones Mathematicae, vol. 14, no. 2, pp. 125-144, 1996.

[4] J.-V. Poncelet, Traité des Propriétés Projectives des Figures: Ouvrage Utile À Qui S'Occupent des Applications de la Géométrie Descriptive et D’opérations Géométriques sur le Terrain, vol. 1-2, Gauthier-Villars, Paris, France, 2nd edition, 1865.

[5] E. W. Weisstein, "Poncelet's Porism," From Math WorldA Wolfram Web Resource, http://mathworld.wolfram.com/ PonceletsPorism.html.

[6] J. Kocik, "Diagram for relativistic addition of velocities," American Journal of Physics, vol. 80, no. 8, p. 720, 2012.

[7] J. Kocik, Interactive diagrams, http://www.math.siu.edu/Kocik/ geometry.html.

[8] J. Cockle, "On certain functions resembling quaternions, and on a new imaginary in algebra," London-Edinburgh-Dublin Philosophical Magazine, vol. 33, pp. 435-439, 1848.

[9] J. Kocik, "Duplex numbers, diffusion systems, and generalized quantum mechanics," International Journal of Theoretical Physics, vol. 38, no. 8, pp. 2221-2230, 1999. 


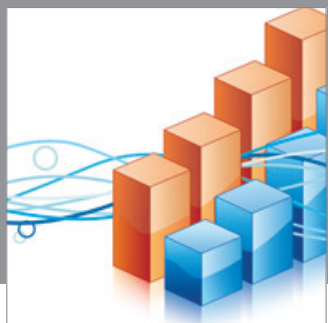

Advances in

Operations Research

mansans

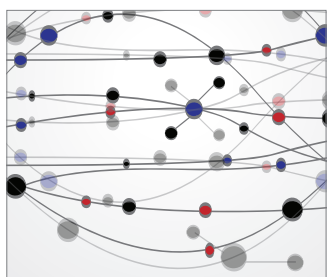

The Scientific World Journal
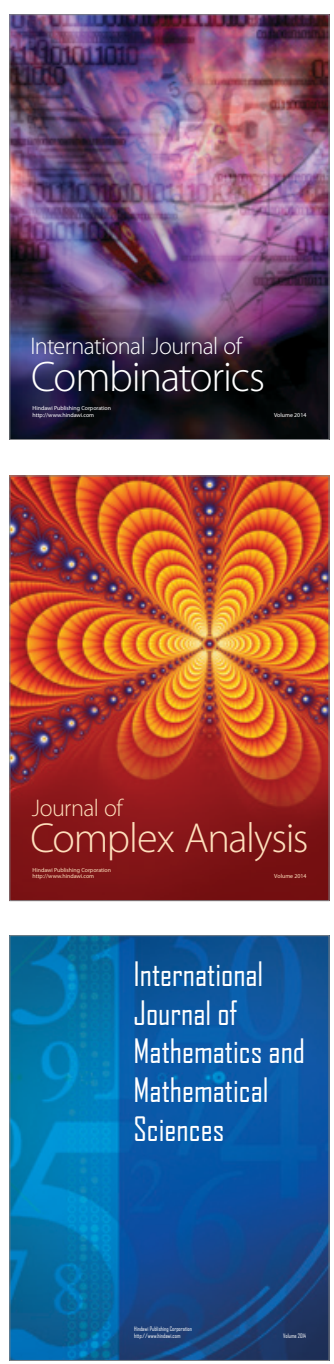
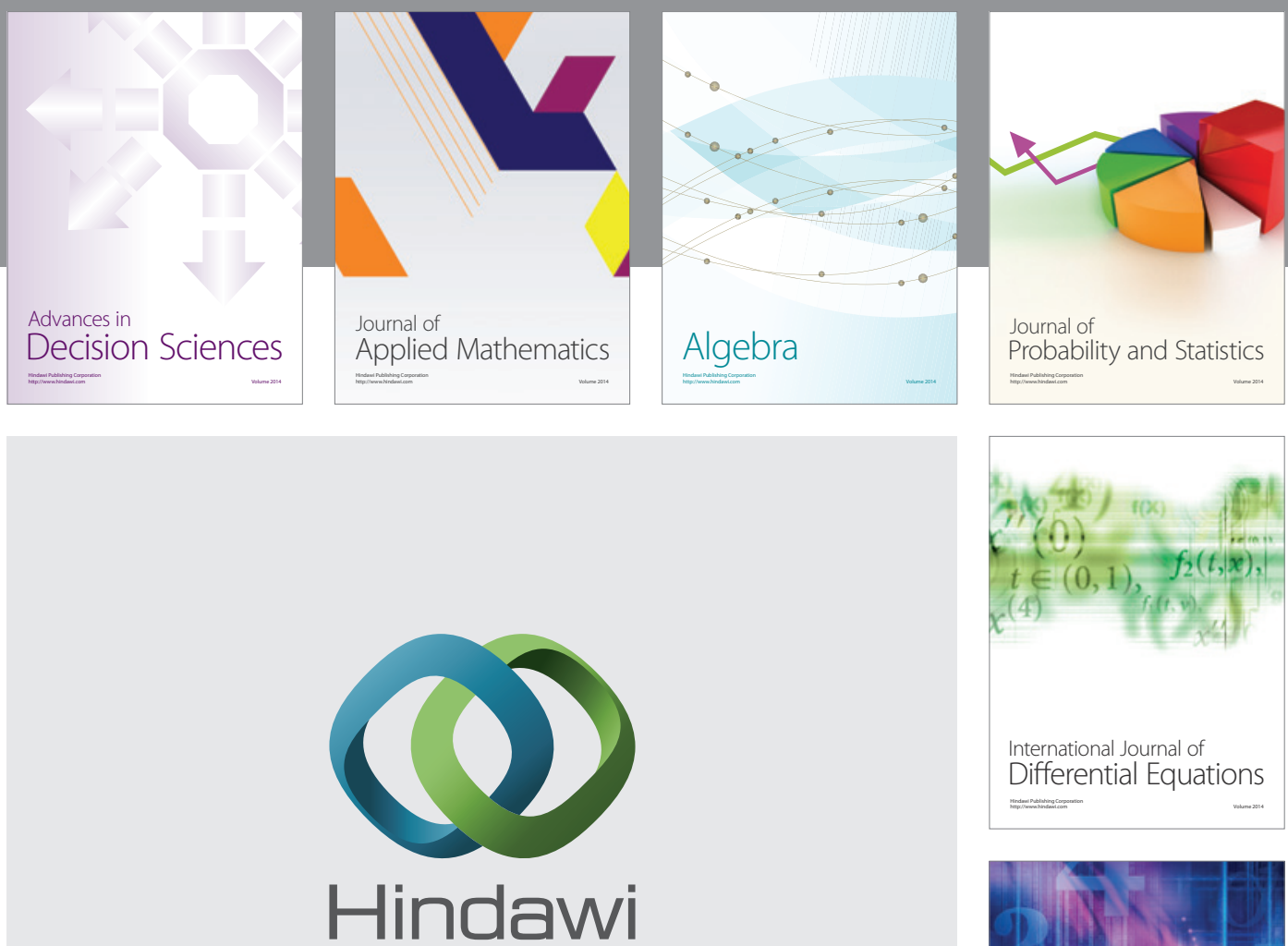

Submit your manuscripts at http://www.hindawi.com
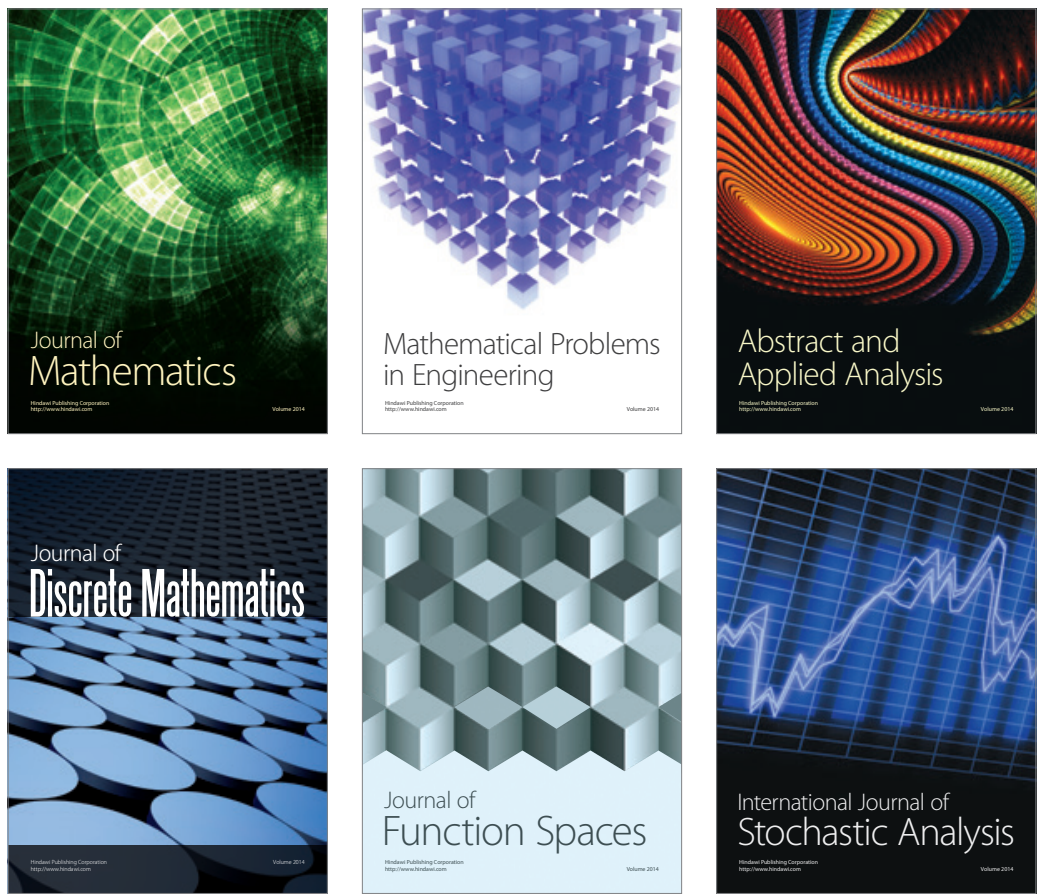

Journal of

Function Spaces

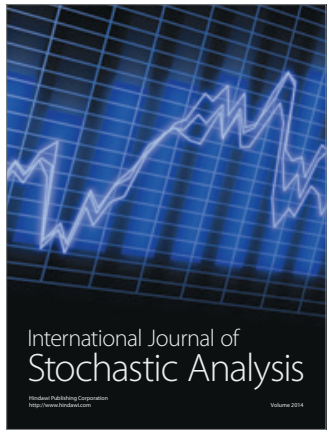

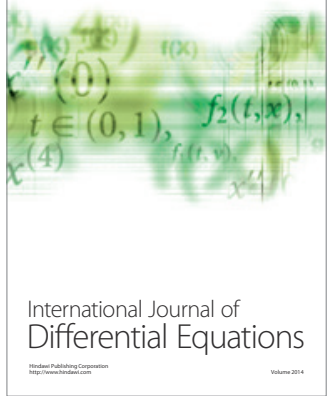
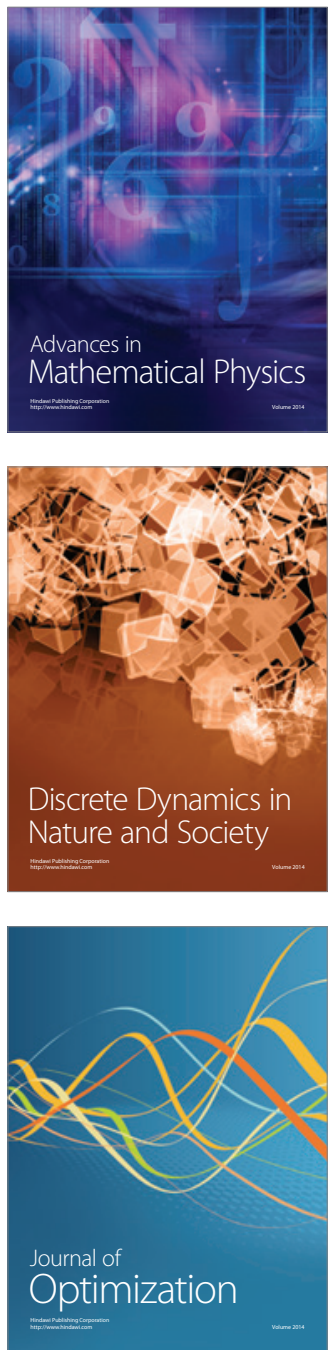